Journal of The Electrochemical Society, 151 (6) A891-A897 (2004)

0013-4651/2004/151(6)/A891/7/\$7.00 @ The Electrochemical Society, Inc.

\title{
Hexagonal to Cubic Spinel Transformation in Lithiated Cobalt
} Oxide

\section{TEM Investigation}

\author{
Heike Gabrisch, ${ }^{\mathrm{a}, *, \mathrm{z}}$ Rachid Yazami, ${ }^{\mathrm{b}, \mathrm{c}}$ and Brent Fultz ${ }^{\mathrm{b}, *}$ \\ ${ }^{a}$ Department of Chemistry, Advanced Materials Research Institute, University of New Orleans, New Orleans, \\ Louisiana 70148, USA \\ ${ }^{b}$ Department of Science and Engineering, California Institute of Technology, Pasadena, \\ California 94702, USA \\ ${ }^{c}$ LEPMI-UMR CNRS 5631, 38402 St. Martin d'Heres, France
}

A transmission electron microscopy (TEM) investigation was performed on $\mathrm{LiCoO}_{2}$ before and after it had been subjected to charge/discharge cycling in electrochemical cells, as well as on delithiated $\mathrm{Li}_{(1-x)} \mathrm{CoO}_{2}$ before and after thermal aging. Turbostratic disorder involving small rotations of the O-Co-O slabs was found in as-received material, and in material subjected to a few cycles. In $\mathrm{LiCoO}_{2}$ subjected to extensive charge/discharge cycling, it was found that increasing amounts of the trigonal $\mathrm{O} 3$ phase had transformed to H1-3 phase and to the cubic spinel phase. The transformation appears to initiate on the surfaces of trigonal crystals. The orientation relationship between the trigonal and spinel phases was determined from diffraction patterns to be $\{0001\}_{\text {trigonal }}$ parallel $\{11\}_{\text {cubic }}$ and $\langle 11 \overline{2} 0\rangle_{\text {trigonal }}$ parallel $\langle 110\rangle_{\text {cubic }}$. The difference in unit cell dimensions leads to transformation stresses when spinel crystals are formed, and spallation of surface layers was observed. The formation of a spinel phase could suppress electrochemical performance of $\mathrm{LiCoO}_{2}$ cathodes in heavily cycled cells. Aging in the charged state also can alter particle surfaces and therefore the performance.

(C) 2004 The Electrochemical Society. [DOI: 10.1149/1.1738677] All rights reserved.

Manuscript submitted June 25, 2003; revised manuscript received January 10, 2004. Available electronically May 4, 2004.

The rechargeable Li-ion batteries in widespread use as power sources for electronic devices are based on the reversible storage of $\mathrm{Li}$ atoms in the crystal structures of the anode and cathode materials. The most commonly used material for positive electrodes in rechargeable $\mathrm{Li}$ batteries is $\mathrm{LiCoO}_{2}$ synthesized at temperatures above approximately $800^{\circ} \mathrm{C}\left(\mathrm{HT}-\mathrm{LiCoO}_{2}\right) .{ }^{1}$ It has a high operating voltage vs. a lithium electrode (3.5-4.2 V) and a large specific capacity $(\approx 140 \mathrm{mAh} / \mathrm{g}){ }^{2}$ Despite its superior properties the capacity in $\mathrm{LiCoO}_{2}$ cathodes decreases with cycle life, an effect that is observed to different extents in all cathodes of rechargeable batteries. The reasons for this failure are not fully understood as of now. The goal of the present study is to monitor microstructural changes occurring in $\mathrm{LiCoO}_{2}$ after large numbers of electrochemical charge-discharge cycles and after aging in the charged state to obtain a better understanding of processes that limit the life of a battery.

The commonly used HT- $\mathrm{LiCoO}_{2}$ belongs into the group of layered $\mathrm{LiMeO}_{2}$ oxides. $^{3}$ It has a trigonal or rhombohedral lattice, consisting of oxygen in $\mathrm{ABC}$ stacking separated by individual layers of $\mathrm{Li}$ and $\mathrm{Co}$ ions. Both types of cations $\left(\mathrm{Co}^{3+}, \mathrm{Li}^{+}\right)$sit in the octahedral interstitial sites between close-packed oxygen layers $(R \overline{3} m$ structure, space group 166, $a=2.82 \AA, c=14.08 \AA$ ) ${ }^{4}{ }^{4}$ This structure is referred to as the $\mathrm{O} 3$ layer structure since $\mathrm{Li}^{+}$ions occupy the octahedral interstitial sites ( $\mathrm{O}$ referring to octahedral), and there are three $\mathrm{MeO}_{2}$ sheets per unit cell, each sheet being formed by edgesharing $\mathrm{CoO}_{2}$ ocathedra. ${ }^{3}$ The strong ionicity of the $\mathrm{Co}-\mathrm{O}$ bonds leads to an important negative charge on the oxygen anions, which destabilizes the layered structure. The cohesive energy of the crystal is lowered by the insertion of $\mathrm{Li}$ cations between the $\mathrm{CoO}_{2}$ slabs. ${ }^{5}$ During charge and discharge cycles, lithium ions are extracted from and reintercalated into the $\mathrm{LiCoO}_{2}$ lattice, causing off-stoichiometric compositions of $\mathrm{Li}_{1-x} \mathrm{CoO}_{2}$.

The changes in the crystal structure of $\mathrm{HT}-\mathrm{Li}_{1-x} \mathrm{CoO}_{2}$ that accompany Li insertion and extraction have been characterized by $\mathrm{X}$-ray and neutron diffraction techniques. In situ X-ray diffraction

\footnotetext{
* Electrochemical Society Active Member.

z E-mail: hgabrisc@uno.edu
}

(XRD) studies have shown that the crystal structure is preserved when the lithium content is cycled between $x=0$ and $x \approx 0.5$. With more Li extraction (larger $x$ ) the interlayer repulsion causes an expansion of the $c$ axis of the unit cell, changing the $c / a$ aspect ratio from 4.99 at full $\mathrm{Li}$ content $(x=0)$ to 5.10 in $\mathrm{Li}_{0.5} \mathrm{CoO}_{2} \cdot{ }^{6}$ At a lithium content $(1-x)$ of 0.5 to 0.45 , however, the lithium ions in the $\mathrm{CoO}_{2}$ framework undergo an order/disorder transition that is coupled to a lattice distortion from the hexagonal to the monoclinic structure. ${ }^{4,7}$ Complete extraction of $\mathrm{Li}$ from the lattice results in the formation of a single-layered hexagonal phase $\mathrm{CoO}_{2}$, the end member of the $\mathrm{Li}_{1-x} \mathrm{CoO}_{2}$ solid solution. Upon reinsertion of $\mathrm{Li}$, this phase converts back to the three-layered, delithiated $\mathrm{Li}_{1-x} \mathrm{CoO}_{2}$ phase. ${ }^{8}$ Fully delithiated $\mathrm{CoO}_{2}$ is believed to be metastable, however, ${ }^{9}$ so the change in stoichiometry should be limited to $\Delta x$ $\approx 0.5$ if acceptable cycle life of the battery it to be obtained $(>500$ cycles). There have been only a few studies on the effect of extensive charge/discharge cycling on crystal structure. ${ }^{10,11}$

At lower synthesis temperatures $\left(400^{\circ} \mathrm{C}\right)$, a different form of $\mathrm{LiCoO}_{2}$ is formed, a cubic spinel phase with compositions denoted $\mathrm{Li}_{1+y} \mathrm{Co}_{2} \mathrm{O}_{4}$. Its oxygen lattice is identical to that of the HT form, but the cation lattice differs. Instead of a separation of Li- and Coions into different $\{111\}$ planes, these planes are now occupied with a mixture of $75 \% \mathrm{Co}$ and $25 \% \mathrm{Li}$, and with $75 \% \mathrm{Li}$ and $25 \% \mathrm{Co}$ on alternating $\{111\}$ planes. $^{12}$ (In more detail, the structure is denoted $F d 3 m$, the space group is $227, \mathrm{Li}$ occupies $16 \mathrm{c}$ sites, Co occupies 16d, O occupies 32 sites with positional parameter $x=0.25$, $a$ $=7.99 \AA$.) This structure is called the lithiated spinel of composition $\mathrm{Li}_{2} \mathrm{Co}_{2} \mathrm{O}_{4}$. This $\mathrm{Li}_{2} \mathrm{Co}_{2} \mathrm{O}_{4}$ transforms to trigonal $\mathrm{LiCoO}_{2}(\mathrm{O} 3$ phase) upon heating. The lithiated spinel is distinguished from the ideal spinel $\mathrm{LiCo}_{2} \mathrm{O}_{4}$ with the $\mathrm{MgAl}_{2} \mathrm{O}_{4}$ structure by the amount of lithium stored in the crystal. In the ideal spinel, lithium is located on tetrahedral 8a sites. The spinel phase was studied as an alternative cathode material because it operates at a lower intercalation voltage, perhaps suppressing decomposition of the electrolyte. The low electroactivity limits its practical application, however. ${ }^{13}$ For electrochemical service, the trigonal layered form (O3 phase) is favorable,${ }^{14}$ whereas the formation of the spinel phase is considered a possible degradation mechanism. ${ }^{10,15}$

Crystallographic studies on these materials have been performed primarily by X-ray and neutron diffractometry. There are, however, 
limitations to these techniques. Rossen et al. ${ }^{13}$ and Gummow et al. ${ }^{16}$ pointed out that it is not possible to distinguish between the layered form of $\mathrm{LiCoO}_{2}$ and the spinel phase from polycrystalline diffraction experiments. Instead the $c / a$ aspect ratio and the electrochemical behavior are used as evidence for either phase. The aspect ratio of the spinel $(c / a=4.914)$ is closer to that of the ideal fcc lattice $(c / a=4.899)$, and varies only little during electrochemical cycling. ${ }^{17}$ The electrochemical signature of the spinel phase is a voltage plateau at $3.7 \mathrm{~V}$ during charge and at $3.5 \mathrm{~V}$ during discharge, compared to $3.9 \mathrm{~V}$ during charge and $3.8 \mathrm{~V}$ during discharge for the trigonal $\mathrm{O} 3$ phase. $^{13}$

The single-crystal diffraction capability of transmission electron microscopy (TEM) can overcome some of the difficulties of powder diffractometry in distinguishing between closely related structures. It is also sensitive to orientation relationships between different crystals, and can provide information on defects and internal strains in crystals. A combination of imaging and diffraction techniques allows the determination of spatial distribution of different phases. Here we present an electron diffraction study of lattice changes in $\mathrm{LiCoO}_{2}$ following extensive electrochemical cycling (334 cycles), as well as thermal aging in the charged state of the battery $(4.2 \mathrm{~V})$. We follow the transformation from the layered $\mathrm{O} 3$ phase to an intermediate H1-3 phase that has been predicted by Van der Ven et al. ${ }^{18}$ to the cubic spinel phase. In additional measurements, $\mathrm{LiCoO}_{2}$ powder was treated chemically to delithiate it in controlled amounts. Electron diffraction showed clearly the coexistence of two phases within heavily cycled crystallites, and showed their orientation relationships.

\section{Experimental}

Virgin $\mathrm{LiCoO}_{2}$ (specimen A), an uncycled cathode prepared from the same material, and a similar cathode subjected to 334 electrochemical charge/discharge cycles (specimen $\mathrm{C}$ ) were provided by courtesy of Enax Inc., Japan. The permanent capacity loss after 334 cycles was in the $10-12 \%$ range. The uncycled $\mathrm{LiCoO}_{2}$ cathode was used to build $\mathrm{Li} / \mathrm{PC}-\mathrm{LiClO}_{4} / \mathrm{LiCoO}_{2}$ coin-type half-cells (CR2016). The uncycled cathode consisted of a composite mixture $(91 \%$ $\mathrm{LiCoO}_{2}, 6 \%$ graphite, and 3\% binder) cast on a thin aluminum foil. The separator was made of glass fibers (Craneglas 230/6.1 from Crane, Co., New Hyde, NY, USA). The electrolyte was $1 \mathrm{M}$ solution of $\mathrm{LiClO}_{4}$ in propylene carbonate (PC) (Mitsubishi Chemicals Co., Tsukuba, Japan). After being mounted in a dry box filled with argon, the cells were subjected to five charge/discharge cycles between 2.9 to $4.2 \mathrm{~V}$ at a C/5 rate at ambient temperature. The cells were then stored at their charged state $\left(\mathrm{Li}_{0.5} \mathrm{CoO}_{2}\right.$ at $\left.4.2 \mathrm{~V}\right)$ at $75^{\circ} \mathrm{C}$ for 10 days. After disassembly of the cells, the $\mathrm{LiCoO}_{2}$ was recovered from the cathode surface by scraping the mixture of oxide, carbon black, and binder off the Al foil and repeatedly washing it in NMP ( $n$ methyl pyrrolidinone) at approximately $40-50^{\circ} \mathrm{C}$ to dissolve the binder (specimen B).

Chemical delithiation was performed by immersing the virgin $\mathrm{LiCoO}_{2}$ powder into an aqueous solution of potassium peroxydisulfate $\left(\mathrm{K}_{2} \mathrm{~S}_{2} \mathrm{O}_{8}\right)$ at $45^{\circ} \mathrm{C}$ for several hours with magnetic stirring. The powder was then washed repeatedly with pure water followed by a wash with methanol, and then dried at $60^{\circ} \mathrm{C}$ in air then in vacuum (specimen D). A portion of the powder was annealed at $300^{\circ} \mathrm{C}$ for several hours (specimen E).

Specimens for routine TEM examination were produced by dispersing a suspension of the powder mixture onto a holey carbon grid. For specimens examined in cross section, the powder in an aged state was embedded in a resin and sliced into thin sections with an ultramicrotome. TEM studies were performed with a Philips EM 420 instrument operated at $120 \mathrm{kV}$. The diffraction studies were accompanied by the simulation of single-crystal electron diffraction patterns using the software package Desktop Microscopist ${ }^{\mathrm{TM}}$ (Lacuna Labs). Appendix A lists all specimens investigated in this study as well as information about their electrochemical behavior. Unit cells used for the simulations are presented in Appendix B. Appendix $\mathrm{C}$ presents the simulated diffraction patterns for lowindex zone axes.

\section{Results}

Results from virgin $\mathrm{LiCoO}_{2}$ (specimen A).--Single-crystal electron diffraction patterns confirmed the $R \overline{3} m$ structure of the trigonal layered $\mathrm{O} 3$ phase of virgin $\mathrm{LiCoO}_{2}$. In the [0001] zone axis, the lowest Bragg reflections (subsequently referred to as diffraction spots or reflections) observed are of $\{11 \overline{2} 0\}$ type corresponding to $1.41 \AA$ lattice spacing (half of the lattice constant, $a$ ). The XRD measurements gave the lattice constant $a=2.82 \AA$.

Moire fringes were observed on many of the virgin $\mathrm{LiCoO}_{2}$ particles. For dark-field imaging, an analysis of the orientation relationship between the fringe direction and active diffraction vector showed that these moiré fringes originated from double diffraction in platelets that were rotated with respect to each other around the $c$-axis (rotation angles $1.5-7^{\circ}$ ). Figure 1a shows a cross section of a crystallite parallel to the $c$-axis, in which separate platelets can be seen. Figure $1 \mathrm{~b}$ shows a plan view of a particle that is covered with moiré fringes, together with the respective diffraction pattern. Out of nine particles analyzed, eight had rotational moiré fringes and one had parallel fringes.

Results from thermally aged material (specimen $B$ ).-The diffraction patterns from $\mathrm{LiCoO}_{2}$ particles aged 10 days at $75^{\circ} \mathrm{C}$ in the delithiated state showed two diffraction features not present from the virgin material: $(i)$ the fundamental $\{11 \overline{2} 0\}$-type reflections were split into two diffraction spots corresponding to two lattice plane spacings $d_{\{h k l\}}$ (Fig. 2a). (ii) New diffraction spots of lesser intensity appeared in the center of triangles formed by the $\{11 \overline{2} 0\}$ reflections (Fig. 2a, b) and halfway between the $\{11 \overline{2} 0\}$ reflections (Fig. 2b).

A comparison with simulated diffraction patterns (Appendix C) allows identification of (a) $\{10 \overline{1} 1\}$-type diffraction spots from the H1-3 phase, (b) \{220\}-type reflections from the cubic spinel phase. The latter reflections are found mostly from material near the particle surface. In some diffraction patterns, we find both the typical diffraction spots of H1-3 and of the spinel phase within the same pattern of fundamental $\{11 \overline{2} 0\}$ reflections, see Fig. $2 b$.

In Fig. 2c, dark-field imaging with a $\{220\}$ reflection of a cubic spinel crystal (Fig. 2b) shows that the spinel phase is located on the particle surface, but not in the interior. Figures $2 b$, c are an image and its corresponding diffraction pattern (taken from the same region of the specimen). To rule out the possibility that the finding of spinel at the particle surface is an artifact of examining a crystal that is electron-transparent at the surface only but not in the center, we prepared one specimen as a cross section by ultramicrotomy. Figure 3 compares microdiffraction patterns from the edge and from the center of the same particle. The particle is shown in the small inset in the respective diffraction patterns. It is seen that near the particle surface, typical reflections of the spinel and H1-3 phase are found (Fig. 3a), whereas the diffraction pattern from the center of the particle corresponds to the original trigonal O3 phase (Fig. 3b).

Results from heavily cycled material (specimen $C$ ).-For the materials subjected to 334 charge/discharge cycles, most diffraction patterns show diffraction spots typical of the spinel phase, and only a few show typical reflections of the H1-3 phase. As in specimen B, we observe the splitting of fundamental $\{11 \overline{2} 0\}_{\text {trigonal-type reflec- }}$ tions into $\{440\}_{\text {cub }}$ and $\{11 \overline{2} 0\}_{\text {trigonal }}$, which indicates that the cubic spinel and the trigonal crystal structures are present. The lattices of both crystals are aligned with parallel planes, $(0001)_{\text {trigl }}$ to $\{111\}_{\text {cub }}$, and parallel directions within the planes: $\langle 11 \overline{2} 0\rangle_{\text {trig }} \|\langle 110\rangle_{\text {cub }}$. Figure $4 \mathrm{a}$ is an example of this orientation re- 


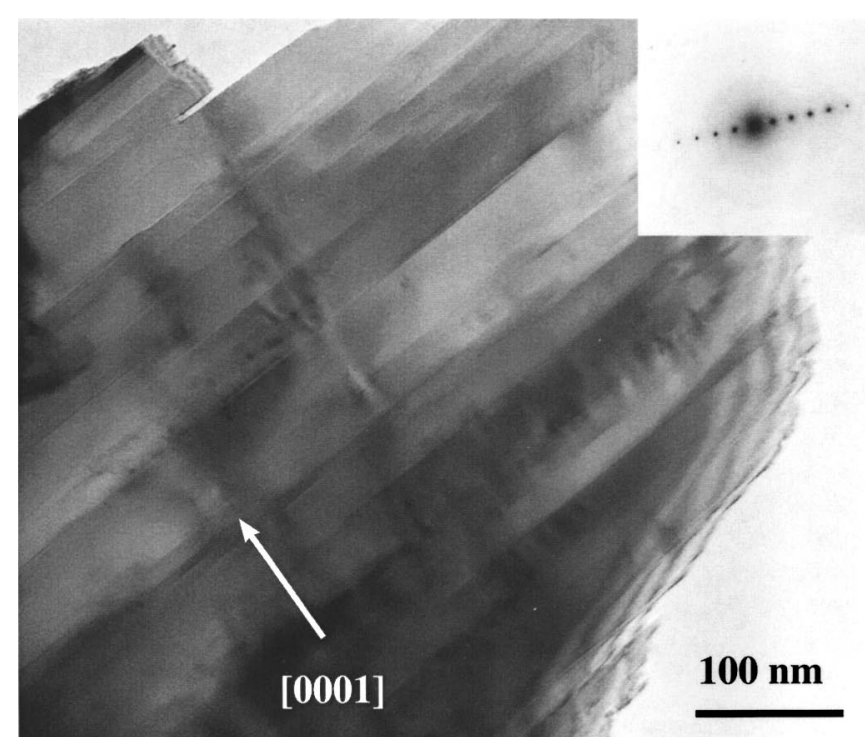

(a)

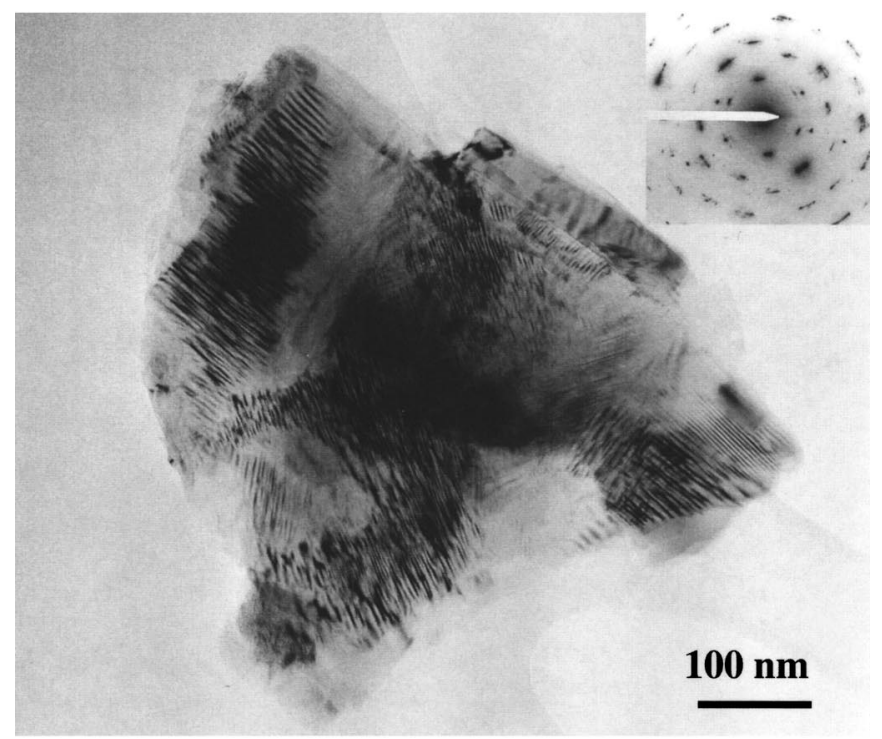

(b)

Figure 1. Virgin $\mathrm{LiCoO}_{2}$. (a) $\mathrm{A}$ cross section of a $\mathrm{LiCoO}_{2}$ particle shows the stacking of crystallites in the $c$ direction. The $c$ axis lies in the image plane. (b) $\mathrm{LiCoO}_{2}$ particle covered with moiré fringes. Analyses of diffraction patterns show that the fringes are of rotational character $(\langle 1 \overline{1} 01\rangle$ zone axis)

lationship, showing a diffraction pattern taken in the $\langle 02 \overline{2} 1\rangle_{\text {trigonal }}$ zone axis, parallel to $\langle 110\rangle_{\text {cubic }}$. The $(\overline{2} 110)$ spot of the trigonal lattice lies adjacent to the (404) fundamental diffraction of the cubic spinel phase (in the same direction, but at a smaller distance in the reciprocal space). The respective lattice constants are $d_{\{440\} \text { cubic }}$ $=1.35 \AA$ and $\mathrm{d}_{\{11 \overline{2} 0\} \text { trigonal }}=1.43 \AA$, a difference of approximately $6 \%$. Since these values were measured from the electron diffraction pattern, the $a$ parameter of the trigonal phase is less reliable than that measured by XRD, but the error is typical of such TEM work. For many crystals, the occurrence of two parallel lattices gives rise to the formation of parallel moire fringes. Such moire fringes can be seen in Fig. 4b taken in the same zone axis, but in a different diffraction condition than Fig. 4a [the active Bragg reflections were $(040)_{\text {cubic }}$ and $\left.(01 \overline{1} \overline{4})_{\text {trigonal }}\right]$. The spacings of par- allel moiré fringes can be used to estimate the difference in lattice parameters of the two crystals. This is found to be approximately $6 \%$, which is agreement with the values obtained directly from the diffraction pattern. The majority of moire patterns in the images of this sample were found to be of parallel character (five of eight). The moiré fringes have a wavy appearance that is superimposed onto a mottled background (Fig. 4b). We believe that inhomogeneous internal strains are responsible for the wavy appearance of the moiré fringes. Frequently we find thick oxide layers spalling off the particle surface, see arrow in Fig. 5. We assume that the difference in lattice constants between the $\mathrm{O} 3$ phase and the spinel phase and the fact that these phases are predominantly located in the particle interior (O3) and on the particle surface (spinel phase) during early stages of cycle life causes strains within the particle that lead to the observed spalling.

Figure 6 shows a dark-field image taken with a $\{220\}$ reflection from the spinel phase. It shows that in this heavily cycled material, the spinel phase is distributed homogeneously throughout the particle covering most of the image. The mottled intensity probably originates with inhomogeneous element distribution. Comparing this image with Fig. 2c suggests that the spinel phase nucleates at the surface and grows into the particle interior with extended cycling or aging.

Results from chemically delithiated and annealed materials (specimens $D, E$ ). - Virgin $\mathrm{LiCoO}_{2}$ subjected to chemical delithiation to $\mathrm{Li}_{0.72} \mathrm{CoO}_{2}$ typically exhibited diffraction patterns from the H1-3 phase. Elemental analysis by ICP-MS reveals an average Li content of 0.72 which leads us to conclude that we see the H1-3 phase and not the $\mathrm{O} 1$ phase, which has a similar diffraction pattern. Dark-field imaging with a $\{10 \overline{1} 1\}$ reflection of the H1-3 phase shows a homogeneous distribution of the phase across one particle. The diffraction pattern shows the same type of splitting of the fundamental $\{11 \overline{2} 0\}$ diffraction spots that was discussed in cycled material as originating with a mixture of trigonal and spinel phases. We found that samples of $\mathrm{Li}_{0.5} \mathrm{CoO}_{2}$ that were heated for several hours at $300^{\circ} \mathrm{C}$ had transformed to the cubic spinel phase.

\section{Discussion}

The transmission electron microscopy (TEM) images are distinctly different for the virgin $\mathrm{LiCoO}_{2}$ and for the $\mathrm{LiCoO}_{2}$ that was subjected to a large number of charge/discharge cycles. The virgin particles show either uniform contrast, or are covered by straight lines of precise moiré fringes (Fig. 1a, b). The heavily cycled particles have a mottled contrast and moiré fringes that are wavy (Fig. 4b, Fig. 6). Furthermore, the nature of the moiré fringes changes from rotational to parallel, indicative of the changes that have taken place in the crystals during cycling.

The presence of rotational moiré fringes, together with a few distinct diffraction patterns with small angles of relative rotation, indicates that in virgin $\mathrm{LiCoO}_{2}$, or material subjected to just a few cycles, there are a few basal planes across which small rotations occur. This rotational disorder probably originates with a relatively weak bonding between $\mathrm{MeO}_{2}$ sheets in the $\mathrm{O} 3$ and $\mathrm{H} 1-3$ structures, and a relatively low cost in energy for a rotational slip across these planes. It is similar to the turbostratic disorder that occurs across the basal planes of graphite. Dislocation analysis revealed the presence of predominantly screw dislocations in the basal planes of virgin $\mathrm{LiCoO}_{2}$ that could account for the observed rotations. ${ }^{19}$ Such deformations could perhaps be growth faults in the virgin material. However, since these rotations are generally small, only a few degrees, it seems more likely that they result from strains in powder preparation when the material is subjected to grinding and/or ballmilling processes to obtain the desired particle size.

After 334 charge/discharge cycles (specimen C), the $\mathrm{LiCoO}_{2}$ was mostly transformed from the layered $\mathrm{O} 3$ phase to the cubic spinel phase. The intensities in our electron diffraction patterns are not 


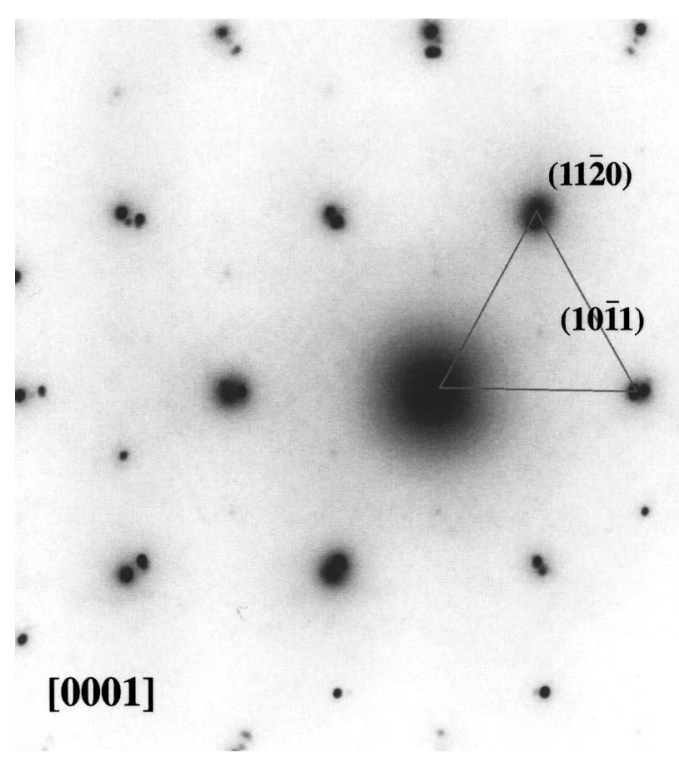

(a)

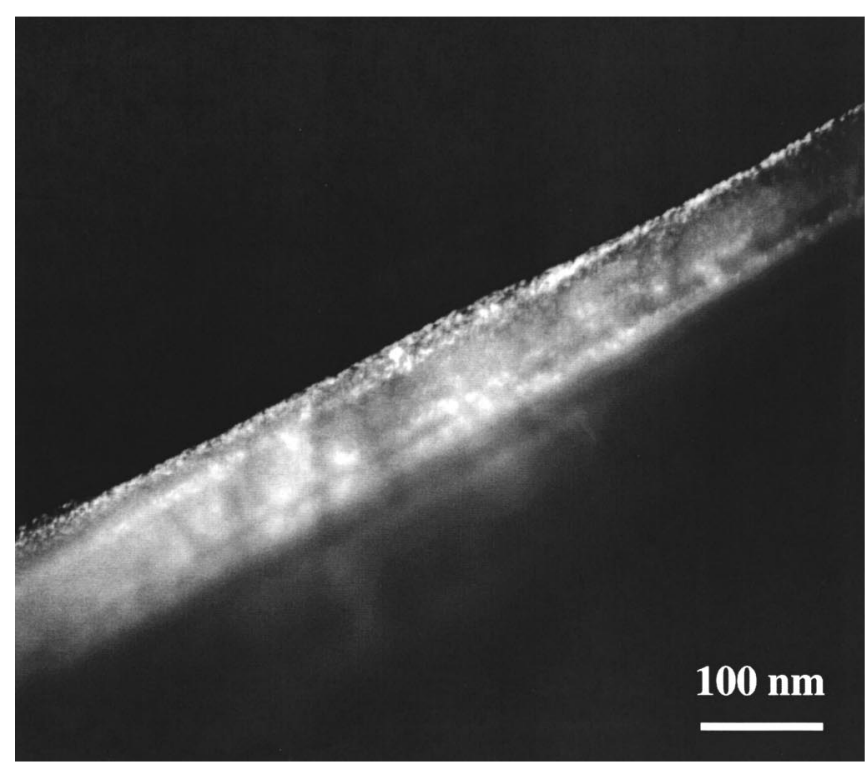

(c)

sufficiently quantitative to distinguish between $\mathrm{Li}$ occupying tetrahedral 8a sites or octahedral 16c sites (ideal spinel vs. lithiated spinel). On the other hand, neutron diffraction has shown that in LT- $\mathrm{LiCoO}_{2}$ that was chemically delithiated by acid treatment to $\mathrm{Li}_{0.5} \mathrm{CoO}_{2}, 80 \%$ of the lithium ions had moved from octahedral $16 \mathrm{c}$ to tetrahedral $8 \mathrm{a}$ sites, producing the ideal spinel structure. ${ }^{17}$ This suggests that the site occupancy depends on the lithium content in the lattice, and suggests that lithium occupies tetrahedral 8a sites in heavily cycled $\mathrm{LiCoO}_{2}$.

In the thermally aged specimen B, both the H1-3 phase and spinel phase were found. The H1-3 phase was predicted by Van der Ven et al. from first-principles theory, ${ }^{18}$ but to our knowledge this is the first experimental evidence for this phase observed in $\mathrm{Li}_{072} \mathrm{CoO}_{2}$ or after cycling between 2.9 and $4.2 \mathrm{~V}$. The finding of the H1-3 phase at $\mathrm{Li}$ concentrations of 0.72 as opposed to much lower Liconcentrations reported in the literature ${ }^{18}$ might be caused by inhomogeneity in the Li concentration of the delithiated specimen. Recently Chen et al. observed the H1-3 phase at a lower Li content after cycling to $4.75 \mathrm{~V}^{20}$ Some particles of specimen B showed both H1-3 and spinel diffraction patterns, whereas in the heavily cycled

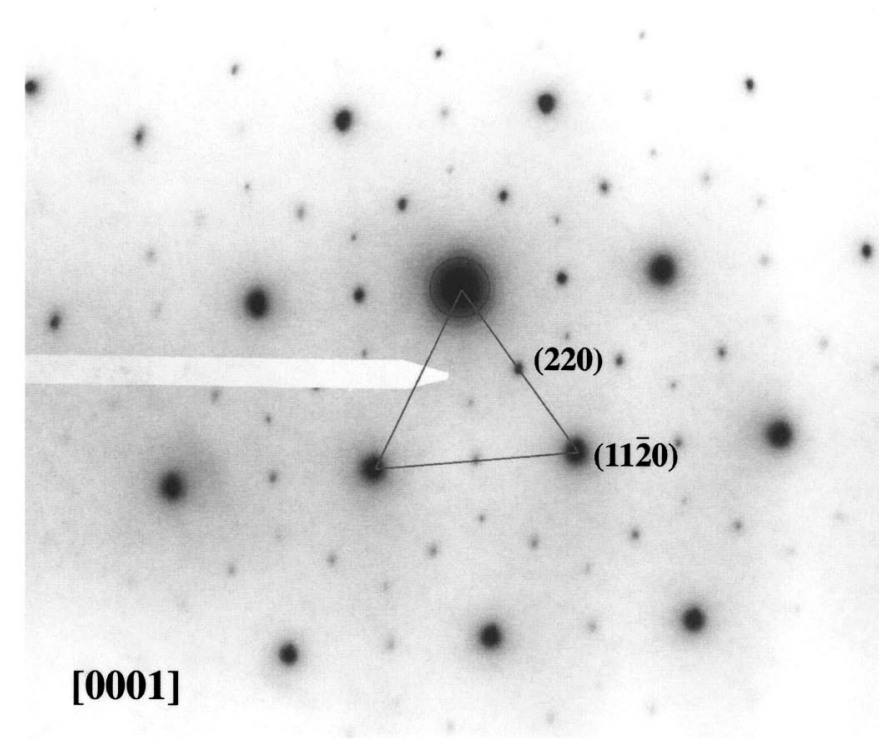

(b)

Figure 2. $\mathrm{LiCoO}_{2}$ cycled five times and annealed at $75^{\circ} \mathrm{C}$ for 10 days. (a) Diffraction pattern of the [0001] zone axis showing the typical diffractions of the H1-3 phase. (b) In some particles a superposition of the H1-3 and spinel diffraction patterns is observed. (c) Dark-field image taken with a $\{220\}$ diffraction from Fig. 2b shows that the spinel phase is located at the particle surface only.

specimen $\mathrm{C}$ there was the mostly spinel phase. As noted previously, the glide of partial dislocations can provide a mechanism for the transformations between the $\mathrm{O} 3, \mathrm{H} 1-3$, and $\mathrm{O} 1$ phases of $\mathrm{Li}_{1-x} \mathrm{CoO}_{2} \cdot{ }^{19}$ The cubic spinel cannot be formed by such crystal shears, and requires diffusional intermixing of the $\mathrm{Li}$ and Co cations. Perhaps the H1-3 phase is an intermediate step in the transformation from the trigonal $\mathrm{O} 3$ to spinel phase of $\mathrm{Li}_{1-x} \mathrm{CoO}_{2}$. In the trigonal H1-3 phase, the environment of the Li-ions changes in every layer from edge-sharing octahedra (O3 phase) to face-sharing octahedra (O1 phase). Face-sharing octahedra provide a path for cation mixing by diffusion (ionic radii in octahedral sites, $\mathrm{Li}^{+}, 0.74 \AA$ 和 ${ }^{3+}, 0.61$ $\AA$ ) which could promote the cation distribution of the spinel phase. This is supported by the following observation on $\mathrm{HT}-\mathrm{LiCoO}_{2}$ that was chemically delithiated to the composition $\mathrm{Li}_{0.5} \mathrm{CoO}_{2}$. This hexagonal phase transformed to the cubic spinel phase upon annealing at $300^{\circ} \mathrm{C}$. Thermodynamic instability of layered $\mathrm{LiCoO}_{2}$ toward spinel was demonstrated through investigations from first principles in Fig. 22 in Ref. 21. 


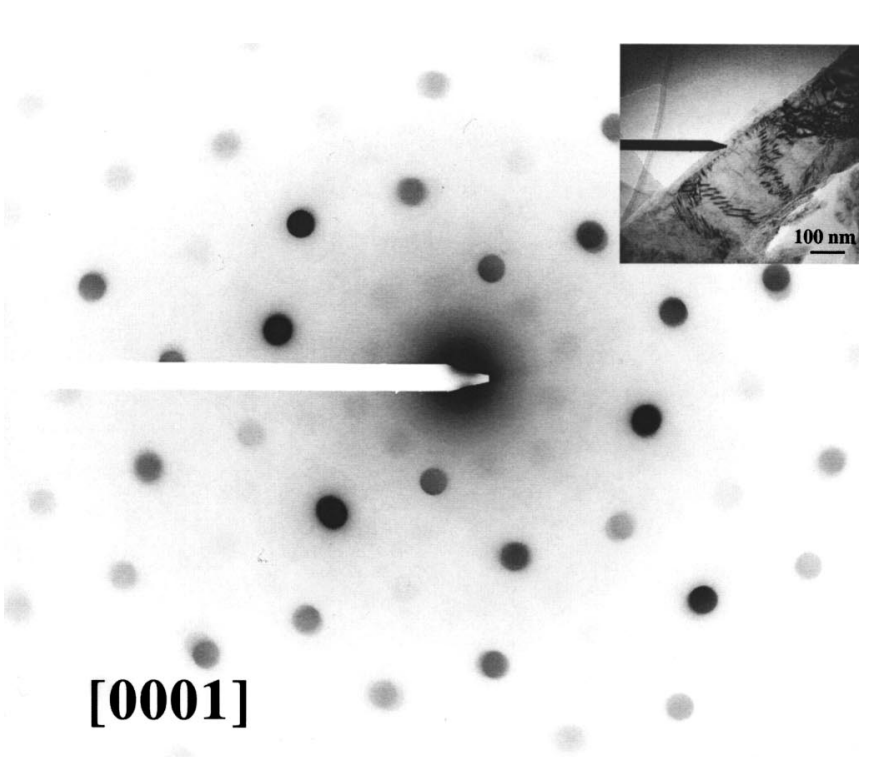

(a)

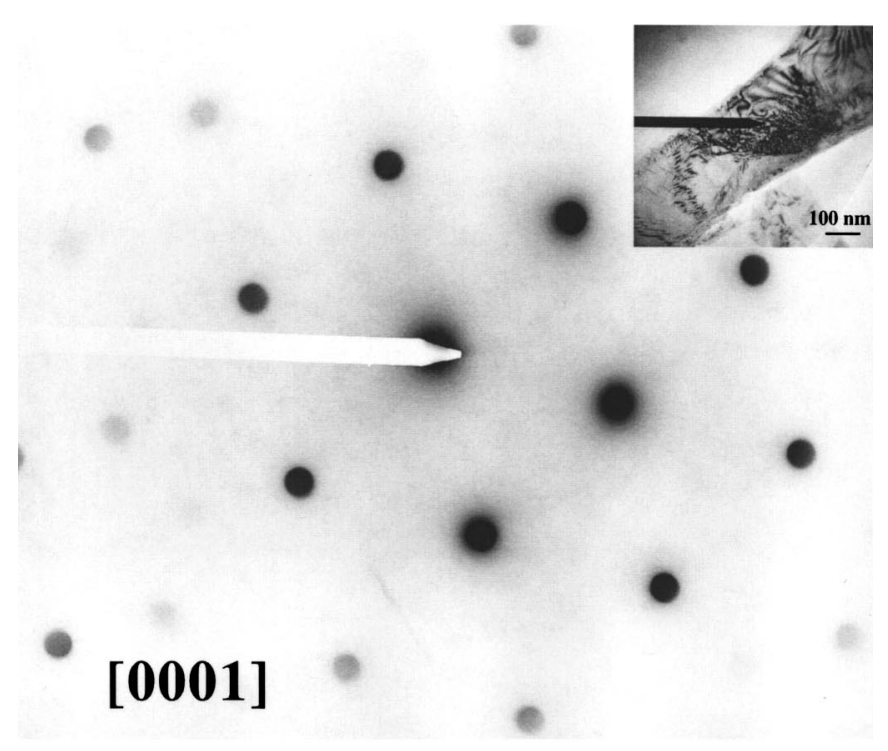

(b)

Figure 3. $\mathrm{LiCoO}_{2}$ cycled five times and annealed at $75^{\circ} \mathrm{C}$ for 10 days. Microdiffraction patterns acquired in the [0001] zone axis from a $\mathrm{LiCoO}_{2}$ specimen prepared by ultramicrotomy. (a) Near the particle surface extra diffractions of the spinel phase are observed. (b) In the particle center only $\{11 \overline{2} 0\}$ diffractions of the trigonal phase are observed.

The separation of the fundamental $\{11 \overline{2} 0\}$ diffraction spots of the original $\mathrm{O} 3$ phase into two diffraction spots is indicative of two aligned crystals. Gummow et al. and Rossen et al. drew a similar conclusion from their measurements of the voltage plateau during $\mathrm{Li}$ extraction from LT- $\mathrm{LiCoO}_{2}$ (lithiated spinel $\mathrm{Li}_{2} \mathrm{Co}_{2} \mathrm{O}_{4}$ ). While they agreed that one of the phases must be the ideal spinel $\mathrm{Li}_{0.5} \mathrm{CoO}_{2}$ they disagreed on the second phase; Gummow suggested it was layered $\mathrm{O} 3$ phase while Rossen suggested it was lithiated spinel $\mathrm{Li}_{2} \mathrm{Co}_{2} 2 \mathrm{O}_{4} \cdot{ }^{12,13}$ Here we can clearly separate between Bragg diffractions originating from the $\mathrm{O} 3$ phase and those originating from the spinel phase (Fig. 4a).

A typical consequence of the transformation of a crystal to another one with a different lattice constant is a mismatch between

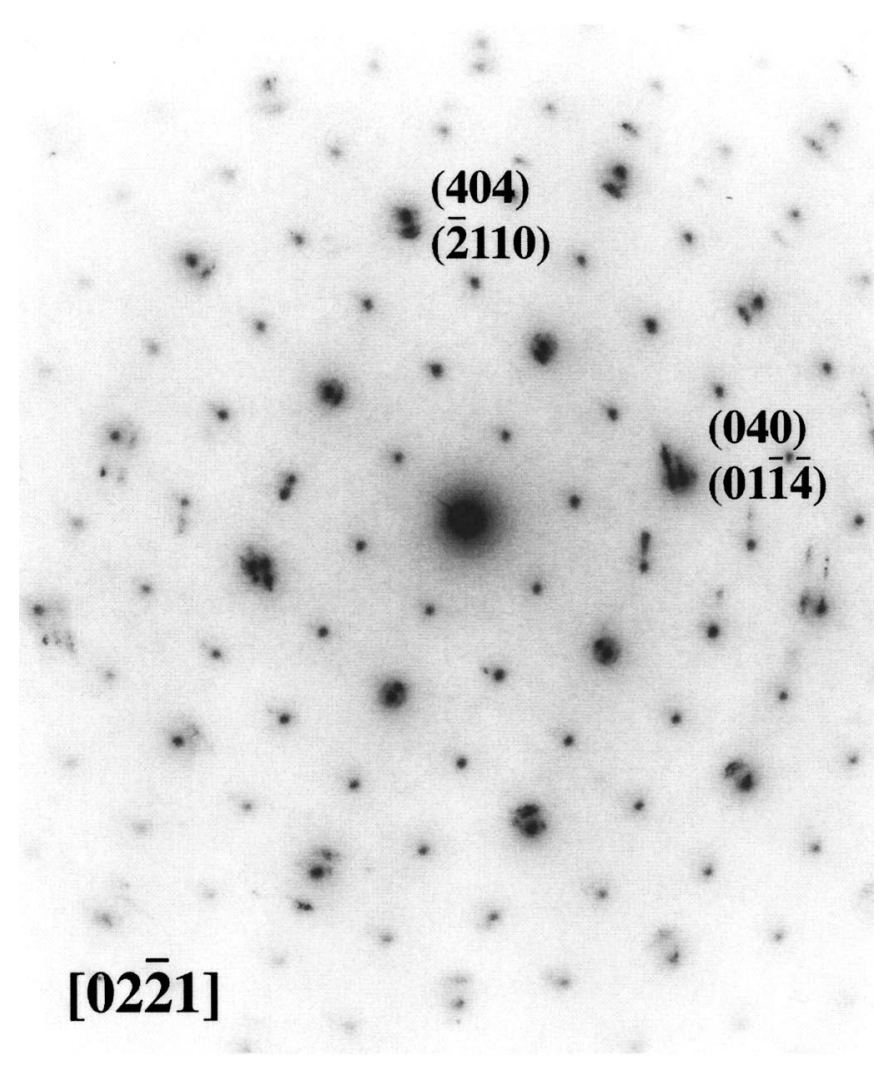

(a)

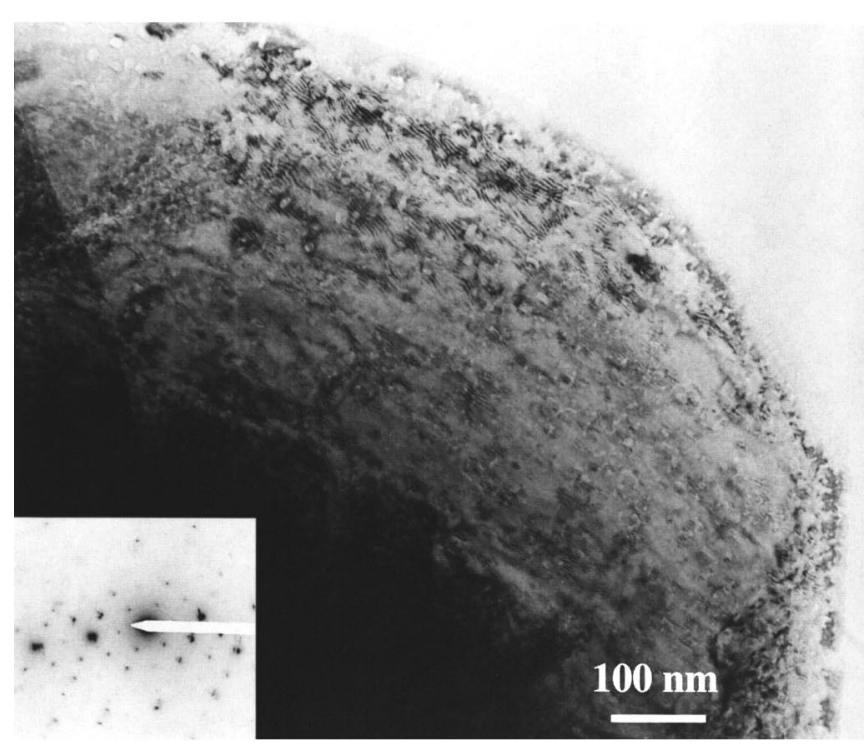

(b)

Figure 4. $\mathrm{LiCoO}_{2}$ cycled 334 times (a) SAD pattern of the [0 $\left.\overline{2} 21\right]$ zone axis showing $\{220\}$ and $\{020\}$ diffractions typical of the cubic spinel phase in addition to $\{11 \overline{2} 0\}$ and $\{0114\}$ diffractions of the trigonal phase. (b) Moiré fringes have a curvy appearance in these specimen, indicative of inhomogeneous strains. Analysis shows that they are of parallel character.

lattice planes in the two crystals. This leads to internal strains from the phase transformation which generate transformation stresses. (Also called Misfit stresses, and often parameterized by a misfit $\delta$ between lattice parameters of two phases, where $\delta=\left(\mathrm{d}_{1}\right.$ $\left.\left.-\mathrm{d}_{2}\right) /\left[0.5\left(\mathrm{~d}_{1}+\mathrm{d}_{2}\right)\right]\right)$. In many particles of cycled material, a spallation of surface layers was observed (see Fig. 5). ${ }^{15}$ These dam- 


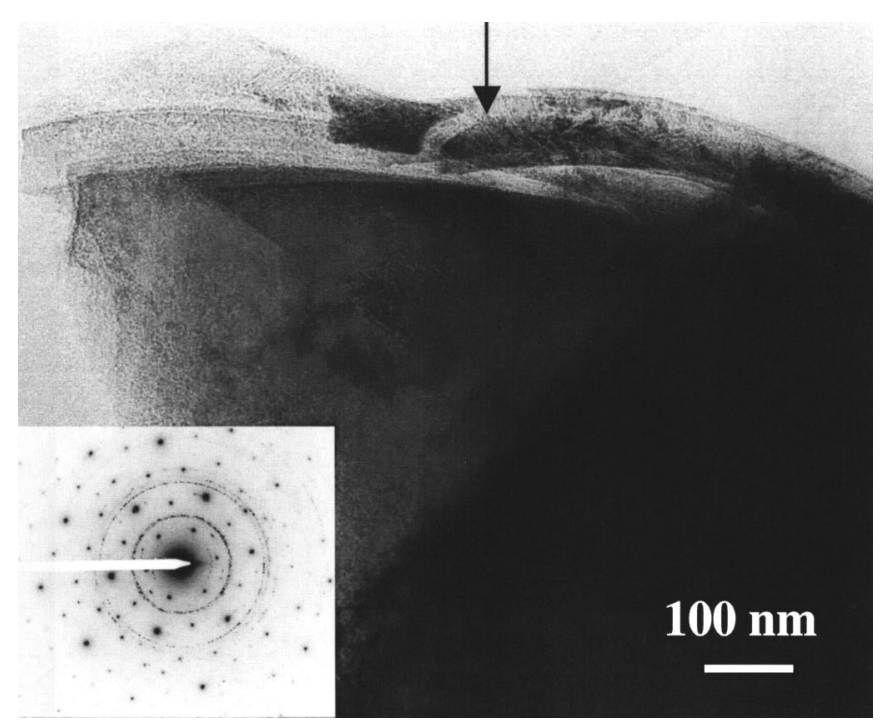

Figure 5. $\mathrm{LiCoO}_{2}$ cycled 334 times. Thick layers of approximately $100 \mathrm{~nm}$ thickness spall off the particle surface (see arrow). The corresponding diffraction pattern taken in [0001] zone axis shows $\{220\}$ diffractions typical of the cubic spinel phase.

aged particles clearly show a diffraction pattern of the spinel phase (see inset in Fig. 5). The degradation of particle surface might contribute to the observed decrease in battery performance. In these damaged areas the conductivity of the interface is interrupted, and active volume is lost for the intercalation of $\mathrm{Li}$. The microstructural arrangement of the spinel and trigonal phases at various stages of cycling is not yet fully understood, however.

All specimen in this study were stable under the electron beam, even at their surfaces. We have no evidence for an organic polymer-

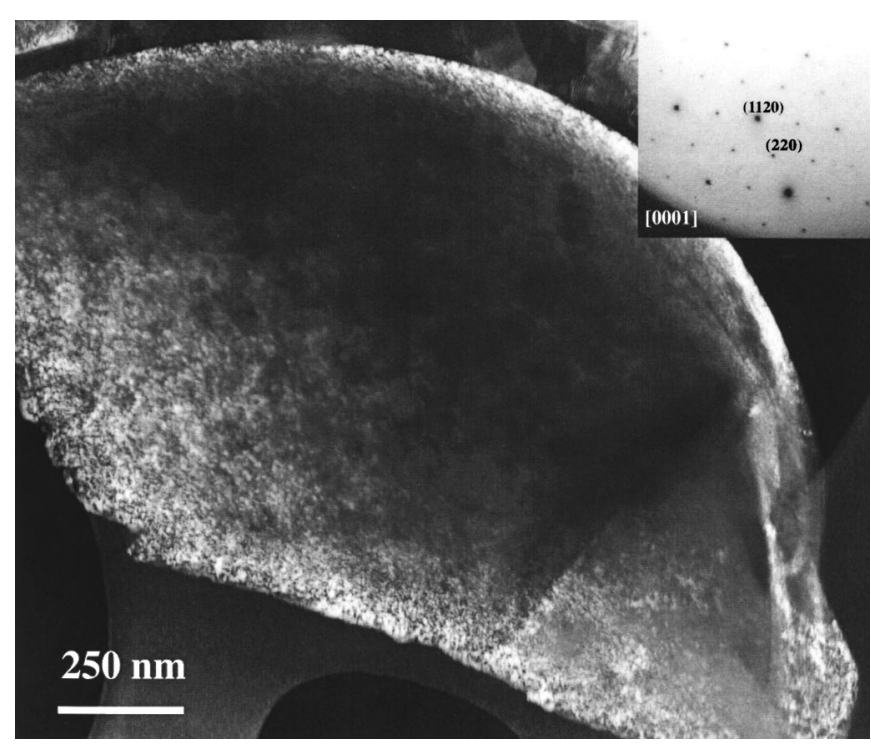

Figure 6. $\mathrm{LiCoO}_{2}$ cycled 334 times. Dark-field imaging with a $\{220\}_{\text {spinel }}$ diffraction demonstrates the homogenous distribution of spinel phase over the whole particle. The corresponding diffraction taken in the [0001] zone axis is shown in the inset. type solid electrolyte interface (SEI) similar to the one on anode surfaces. ${ }^{22}$ In $\mathrm{LiCoO}_{2}$ the only observable surface change was the formation of the cubic spinel phase. Although the fractional amount of the spinel phase is often small, it is significant that this phase is found on the surface of the layered $\mathrm{O} 3$ phase of $\mathrm{LiCoO}_{2}$. A continuous layer of spinel could alter the kinetics of lithiation and delithiation. If sufficiently thick, a layer of the spinel phase could suppress the effective electrochemical capacity of the $\mathrm{LiCoO}_{2}$ by more than its fractional amount.

\section{Conclusions}

In virgin $\mathrm{LiCoO}_{2}$ with the $\mathrm{O} 3$ trigonal crystal structure, rotational moiré patterns in the images and rotations in the single-crystal electron diffraction patterns indicated a turbostratic disorder from small rotations of the O-Co-O slabs. These rotations could be induced by stresses in crystal growth or stresses resulting from mechanical treatment during powder production. $\mathrm{In} \mathrm{LiCoO}_{2}$ subjected to extensive charge/discharge cycling, the analysis of single-crystal electron diffraction patterns showed a transformation of parts of the trigonal $\mathrm{LiCoO}_{2}$ into a delithiated spinel $\mathrm{Li}_{1+y} \mathrm{Co}_{2} \mathrm{O}_{4}$. The spinel phase forms first at the surfaces of crystals, but eventually extends throughout some crystals. The orientation relationship between the two phases was determined from diffraction patterns to be $\{0001\}_{\text {trigonal }}$ parallel $\{111\}_{\text {cubic }}$ and $\langle 11 \overline{2} 0\rangle_{\text {trigonal }}$ parallel $\langle 110\rangle_{\text {cubic }}$. The difference in unit cell dimensions leads to transformation stresses when spinel crystals are formed. The subsequent mechanical degradation of the particle includes spallation of surface layers. The reduced conductivity and loss of active volume as well as the lower electrochemical activity of the spinel phase are possible reasons for the observed decrease in electrochemical performance of $\mathrm{LiCoO}_{2}$ cathodes.

\section{Acknowledgments}

The authors thank Yasunori Ozawa for preparing the thermally aged specimen, Dr. Jason Graetz for preparing the chemically delithiated specimen, Enax Co. (Japan) for providing virgin and heavily cycled $\mathrm{LiCoO}_{2}$. This work was supported by the U.S. DOE through Basic Energy Sciences grant DE-FG03-00ER15035.

California Institute of Technology assisted in meeting the publication costs of this article.

\section{Appendix A \\ Specimen Treatment}

The following specimen have been investigated in this study:

Virgin $\mathrm{LiCoO}$. - Pristine $\mathrm{LiCoO}_{2}$ powder, X-ray diffraction (not shown here) gave $a=2.817 \AA$ and $c=14.03 \AA$ as unit cell parameters of the hexagonal phase.

Thermally aged.-Sample A-based electrode comprised of wt $\% 91 \mathrm{LiCoO}_{2}$, wt $\%$ 6 graphite, and wt \% 3 PVDF binder. A lithium half-cell battery was made of it using 1 $\mathrm{M} \mathrm{LiClO}_{4}$ in PC as the electrolyte. The cell was cycled five times then charged to $4.2 \mathrm{~V}$ and annealed at $75^{\circ} \mathrm{C}$ for 10 days.

Heavily-cycled.-Sample B electrode taken from a lithium-ion prototype battery cycled 334 times between 3.0 and $4.2 \mathrm{~V}$ under $1 \mathrm{C} / 1 \mathrm{C}$ rate at ambient temperature. The final state of the battery was a discharge to $3.0 \mathrm{~V}$. The battery had experienced a permanent capacity loss of $10-12 \%$.

Chemically delithiated.-Sample A treated with a $\mathrm{K}_{2} \mathrm{~S}_{2} \mathrm{O}_{8}$ solution to remove 0.28 $\mathrm{Li} /$ mole. The powder is then repeatedly washed with methanol and dried in vacuum at $50-60^{\circ} \mathrm{C}$ for $12-24 \mathrm{~h}$.

Chemically delithiated and annealed. $-\mathrm{Li}_{0.5} \mathrm{CoO}_{2}$ annealed at $300^{\circ} \mathrm{C}$ in vacuum for several hours. 


\section{Appendix B}

Crystallographic Unit Cells

Trigonal unit cell, Ref. 4.

Space group 166, $R \overline{3} m a=2.82 \AA, c=14.06 \AA$

Atom Wyckoff position

\begin{tabular}{lll}
$\mathrm{Li}$ & $3 \mathrm{a}$ & $(0,0,0)$ \\
$\mathrm{Co}$ & $3 \mathrm{~b}$ & $(0,0,0)$ \\
$\mathrm{O}$ & $6 \mathrm{c}$ & $(0,0,0.25)$ \\
\hline
\end{tabular}

H1-3 unit cell, Ref. 29 in 18.

Space group 166, $R \overline{3} m a=2.78 \AA, c=25.77 \AA$

Atom Wyckoff position

\begin{tabular}{lll}
$\mathrm{Li}$ & $3 \mathrm{a}$ & $(0,0,0)$ \\
$\mathrm{Co}$ & $6 \mathrm{c}$ & $(0,0,0.42)$ \\
$\mathrm{O}$ & $6 \mathrm{c}$ & $(0,0,0.29)$ \\
$\mathrm{O}$ & $6 \mathrm{c}$ & $(0,0,0.12)$ \\
\hline
\end{tabular}

Spinel unit cell, Ref. 16.

Space group 227, $F d 3 m a=8.02 \AA$

$\mathrm{Li}_{2} \mathrm{Co}_{2} \mathrm{O}_{4}$

Atom Wyckoff position

\begin{tabular}{lll}
$\mathrm{Li}$ & $16 \mathrm{c}$ & $(0,0,0)$ \\
$\mathrm{Co}$ & $16 \mathrm{~d}$ & $(0.5,0.5,0.5)$ \\
$\mathrm{O}$ & $32 \mathrm{e}$ & $(0.26,0.26,0.26)$ \\
\hline
\end{tabular}

$a=8.02 \AA$

$\mathrm{LiCo}_{2} \mathrm{O}_{4}$

Atom Wyckoff position

$\begin{array}{lll}\mathrm{Li} & 8 \mathrm{a} & (0.125,0.125,0.125) \\ \mathrm{Co} & 16 \mathrm{~d} & (0.5,0.5,0.5) \\ \mathrm{O} & 32 \mathrm{e} & (0.26,0.26,0.26)\end{array}$

Appendix C

Simulations of Diffraction Patterns

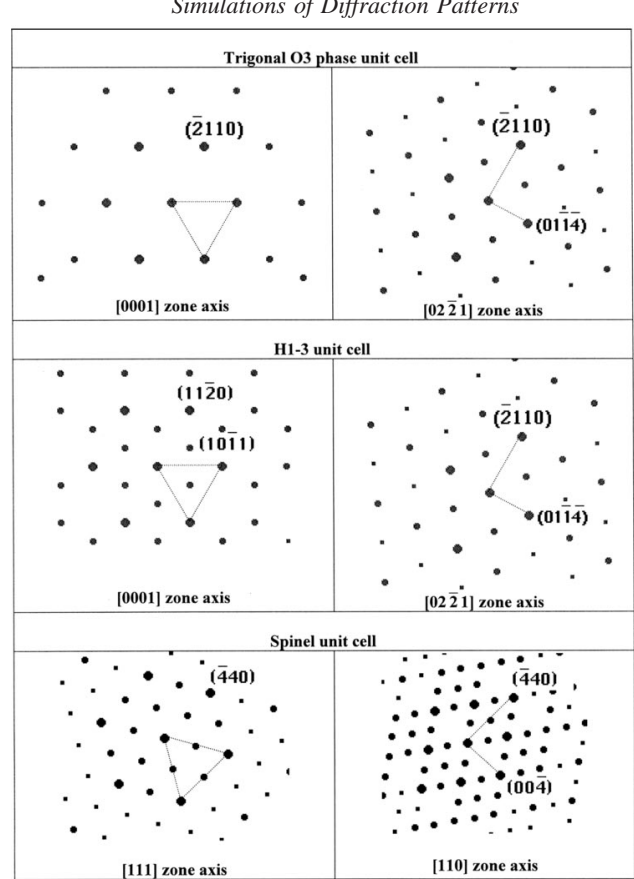

References

1. A. Honders, J. M. der Kinderen, A. V. van Heeren, J. H. W. de Wit, and G. H. J. Broers, Solid State Ionics, 15, 265 (1985).

2. J. B. Goodenough, in Lithium Ion Batteries-Fundamentals and Performance, M. Wakihara and O. Yamamoto, Editors, p. 1, Wiley-VCH, Weinheim (1998).

3. A. Manthiram, in Lithium Batteries, G. A. Nazri and G. Pistoia, Editors, Kluwer Academic Publishers, New York (2003).

4. J. N. Reimers and J. R. Dahn, J. Electrochem. Soc., 139, 2091 (1992).

5. C. Delmas in Lithium Batteries-New Materials, Developments and Perspectives, G. Pistoia, Editor, Chap. 12, Elsevier, New York (1994).

6. T. Ohzuku and A. Ueda, J. Electrochem. Soc., 141, 2972 (1994).

7. K. Mitzushima, P. C. Jones, P. J. Wiseman, and J. P. Goodenough, Mater. Res. Bull. 15, 783 (1980).

8. G. G. Amatucci, J. M. Tarascon, and L. C. Klein, J. Electrochem. Soc., 143, 1114 (1996).

9. M. Broussely, P. Biensan, and B. Simon, Electrochim. Acta, 45, 3 (1999)

10. H. Wang, Y.-I. Jang, B. Huang, D. R. Sadoway, and Y.-M. Chiang, J. Electrochem. Soc., 146, 473 (1999)

11. J. Li, E. Murphy, and P. A. Kohl, J. Power Sources, 102, 294 (2001).

12. R. J. Gummow, M. M. Thackeray, W. I. F. David, and S. Hull, Mater. Res. Bull., 27, 327 (1992).

13. E. Rossen, J. N. Reimers, and J. R. Dahn, Solid State Ionics, 62, 53 (1993)

14. G. Ceder, A. Van der Ven, and M. K. Aydinol, J. Miner, Met, Mater, 50, 35 (1998).

15. H. Gabrisch, R. Yazami, and B. Fultz, J. Power Sources, 119-121, 674 (2003),

16. R. J. Gummow, D. C. Liles, and M. M. Thackeray, Mater. Res. Bull., 28, 235 (1993).

17. R. J. Gummow, D. C. Liles, M. M. Thackeray, and W. I. F. David, Mater. Res. Bull. 28, 1177 (1993).

18. A. Van der Ven, M. K. Aydinol, and G. Ceder, Phys. Rev. B, 58, 2975 (1998).

19. H. Gabrisch, R. Yazami, and B. Fultz, Electrochem. Solid-State Lett., 5, A111 (2002).

20. Z. Chen, Z. Lu, and J. R. Dahn, J. Electrochem. Soc., 149, A1604 (2002).

21. G. Ceder and A. Van der Ven, Electrochim. Acta, 45, 131 (1999).

22. R. Yazami, in Li-batteries, New Materials, Developments and Perspectives, G. Pistoia, Editors, p. 49, Elsevier, New York (1994). 